\title{
छூ \\ Dielectric laser acceleration of nonrelativistic electrons at a single fused silica grating structure: Experimental part
}

\author{
John Breuer, Roswitha Graf, ${ }^{\dagger}$ and Alexander Apolonski ${ }^{\ddagger}$ \\ Max Planck Institute of Quantum Optics, Hans-Kopfermann-Straße 1, 85748 Garching, Germany \\ Peter Hommelhoff \\ Department of Physics, Friedrich Alexander University Erlangen Nuremberg, \\ Staudtstr. 1, 91058 Erlangen, Germany and Max Planck Institute of Quantum Optics,
}

Hans-Kopfermann-Straße 1, 85748 Garching, Germany

(Received 23 October 2013; published 6 February 2014)

\begin{abstract}
Recently, our group has demonstrated dielectric laser acceleration of nonrelativistic electrons at a scalable fused silica grating [J. Breuer and P. Hommelhoff, Phys. Rev. Lett. 111, 134803 (2013)]. This represents a demonstration of the inverse Smith-Purcell effect in the optical regime. The third spatial harmonic of the grating, which is excited by Titanium:sapphire laser pulses, synchronously accelerates $28 \mathrm{keV}$ electrons derived from an electron microscope column. We observe a maximum acceleration gradient of $25 \mathrm{MeV} / \mathrm{m}$. Here we present the experimental setup in detail. We describe grating-related issues such as surface charging and alignment as well as damage threshold measurements. A detailed explanation of the detection scheme is given. Furthermore, extensive numerical simulations are discussed, which agree well with the experimental results.
\end{abstract}

DOI: 10.1103/PhysRevSTAB.17.021301

PACS numbers: 41.75.Jv, 42.25.-p, 42.50.Wk

\section{INTRODUCTION}

Laser-driven particle acceleration has been proposed just two years after the realization of the first lasers because it was clear that well-controlled and large optical field strength could be achieved [1]. Hence laser-driven particle acceleration bears the promise of high acceleration gradients and may therefore lead to much smaller accelerators than achievable with conventional radio frequency (rf) linacs, which currently operate with an acceleration gradient of $\sim 20-50 \mathrm{MeV} / \mathrm{m}$. The maximum achievable acceleration gradients are ultimately limited by the damage threshold of the materials that are used to confine the accelerating fields. State-of-the-art accelerators, based on rf technology, will not operate far beyond $100 \mathrm{MeV} / \mathrm{m}$ due to breakdown phenomena at the metal structures $[2,3]$. Because of the 1-2 orders of magnitude larger damage threshold of dielectrics at optical frequencies [4] compared to metals at microwave frequencies, as well as the rapid

\footnotetext{
*Present address: Integrated Circuit Testing GmbH, Ammerthalstraße 20, 85551 Heimstetten, Germany.

Present address: AMPHOS GmbH, Kaiserstraße 100, 52134 Herzogenrath, Germany.

${ }^{\ddagger}$ Also at: Ludwig-Maximilians-Universität München, Am Coulombwall 1, 85748 Garching, Germany.

peter.hommelhoff@fau.de

Published by the American Physical Society under the terms of the Creative Commons Attribution 3.0 License. Further distribution of this work must maintain attribution to the author(s) and the published articles title, journal citation, and DOI.
}

advances in controlling ultrashort laser pulses over the last two decades [5], dielectric laser accelerators have been proposed to drive the next generation linac with acceleration gradients of beyond $1 \mathrm{GeV} / \mathrm{m}$ [6-11].

For sustainable particle acceleration with oscillating electromagnetic fields, an electromagnetic wave with a phase speed equal to and an electric field component parallel to the particle's velocity is required, which continuously imparts energy to the particle. Direct laser acceleration in a vacuum with the longitudinal field component inside the tight focus of a laser beam is possible and has recently been observed [12], but due to the mismatch of the phase velocity of the accelerating field and the particle's velocity, the acceleration is confined to approximately the Rayleigh length of the laser beam $[13,14]$. Very large acceleration gradients on the order of tens of $\mathrm{GV} / \mathrm{m}$ have been demonstrated with laser-driven plasma wakefield acceleration [15]. In this scheme the particles are accelerated by a plasma wave that is excited by an intense $\left(>10^{18} \mathrm{~W} / \mathrm{cm}^{2}\right)$ laser pulse.

The first demonstration of laser-driven acceleration in the vicinity of a dielectric material has been reported in $[16,17]$. Here relativistic electrons could be accelerated by up to $40 \mathrm{MeV} / \mathrm{m}$. However, the reported scheme lacks the possibility of straightforward concatenation, i.e., scalability.

Surface waves that can exist close to periodic grating structures can be used for synchronous and scalable vacuum acceleration $[18,19]$. This near-field-based acceleration scheme is also known as the inverse Smith-Purcell effect [20]. It has been demonstrated with a terahertz 
radiation source $[21,22]$ at a metal grating of $250 \mu \mathrm{m}$ period, but acceleration gradients were too small $(\mathrm{keV} / \mathrm{m})$ to compete with rf accelerators. Our proof-of-concept experiment exploits this effect in the vicinity of a dielectric grating structure. The difference to the originally proposed inverse Smith-Purcell effect is that here the grating modes are excited in transmission as opposed to reflection [20].

The dielectric grating used in our experiment is directly compatible with the double grating structures proposed by Plettner et al. [9,23-26]. Recently, dielectric laser acceleration of relativistic electrons has been observed at SLAC [27], in parallel with our demonstration of nonrelativistic electron acceleration at a similar structure [28]. Because of the intercompatibility of the two experiments, an all-optical laser-driven accelerator, including nonrelativistic and relativistic sections, seems now feasible. In this paper we present a detailed overview of our experiment, including setup, simulations, and results.

\section{THEORY}

Particle acceleration at a grating is based on the diffraction of laser light oscillating with a frequency $f=c / \lambda$, with $c$ the speed of light and $\lambda$ the laser wavelength. The diffracted light field is associated with evanescent modes, also known as spatial harmonics, in close vicinity of the grating surface. The $n$th spatial harmonic is characterized by $n$ oscillations per grating period $\lambda_{\mathrm{p}}[n=3$ in Figs. 1 $(\mathrm{a}-\mathrm{c})$ ]. Therefore, it propagates along the grating surface with a phase velocity $v_{\mathrm{ph}}=f \lambda_{\mathrm{p}} / n=c \lambda_{\mathrm{p}} /(n \lambda)$. Hence, particles with the velocity $v=\beta c=v_{\text {ph }}$ can surf on and continuously interact with this synchronous mode. This yields the synchronicity condition [25]

$$
\beta=\frac{\lambda_{\mathrm{p}}}{n \lambda} .
$$

By solving the wave equation $\left(c^{2} \nabla^{2}-\partial^{2} / \partial t^{2}\right) \vec{E}(\vec{r}, t)=0$, it can be shown that the field strength falls off exponentially with increasing distance from the grating surface with a decay constant

$$
\Gamma=\frac{\beta \gamma \lambda}{2 \pi},
$$

with $\gamma=1 / \sqrt{1-\beta^{2}}$ [26]. Because of the field geometry of the synchronous mode the particle can experience acceleration, deceleration, or deflection, depending on its relative position inside the field [Figs. 1(a-c)]. The effect of all other asynchronous spatial harmonics averages to zero over time.

As nonrelativistic electrons significantly change speed during the acceleration they may run out of phase and eventually become decelerated again. Therefore, in future experiments the grating period needs to be adaptively
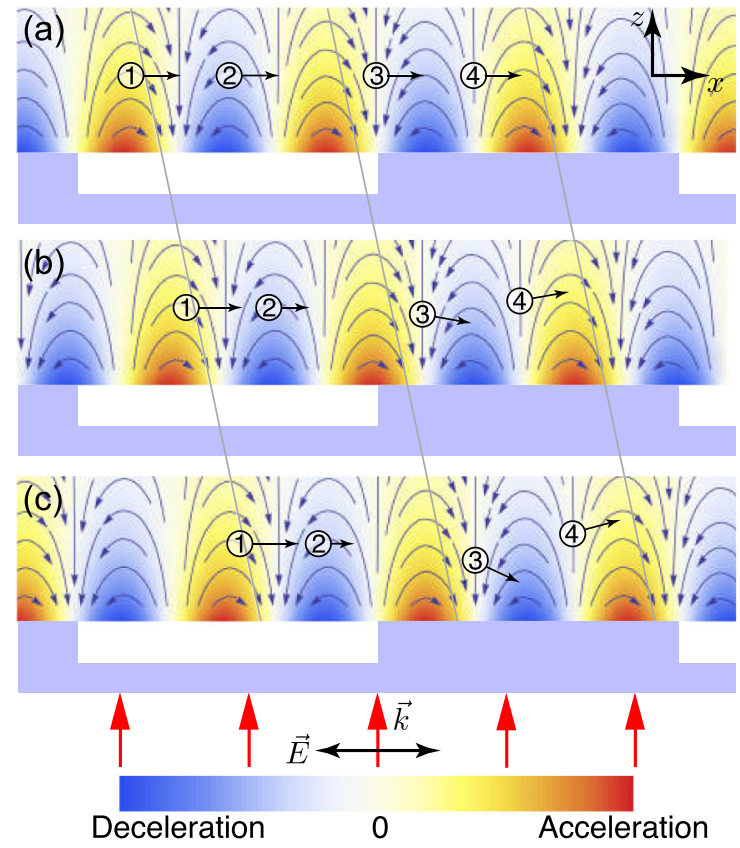

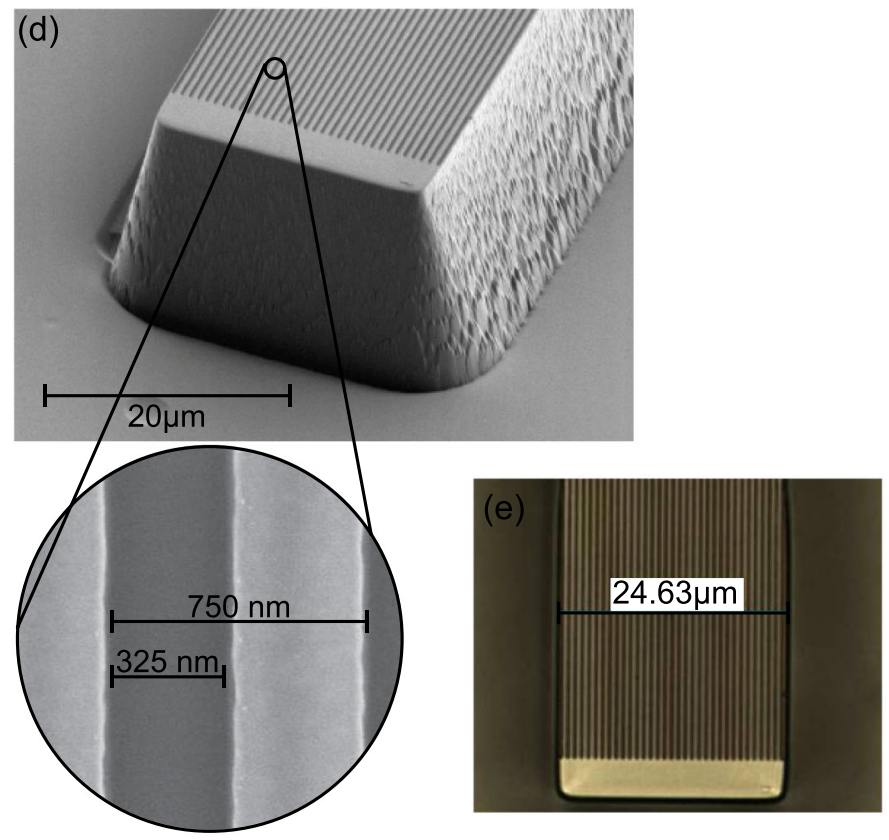

FIG. 1 (color online). (a-c) Three consecutive snapshots in time (a quarter optical period apart) of the electric field distribution of the third spatial harmonic (blue arrows) above a grating (light blue structure). This surface wave, excited by a linearly polarized laser from below (red arrows), propagates synchronously with the charged particles (numbered circles) along the grating surface. Here the charged particles are assumed to be positrons. Depending on the relative position of the positron inside the field the force can lead to either acceleration (1), deceleration (2), or deflection $(3,4)$, as indicated by the blue arrows and the color shading. (d) Electron microscope image of the fused silica grating that is located on top of a mesa, $20 \mu \mathrm{m}$ above the substrate. The closeup shows the grating with a grating period $\lambda_{\mathrm{p}}=750 \mathrm{~nm}$, a trench width of $325 \mathrm{~nm}$, and a depth of $280 \mathrm{~nm}$. (e) Top view of the mesa with a width of $25 \mu$ m. 


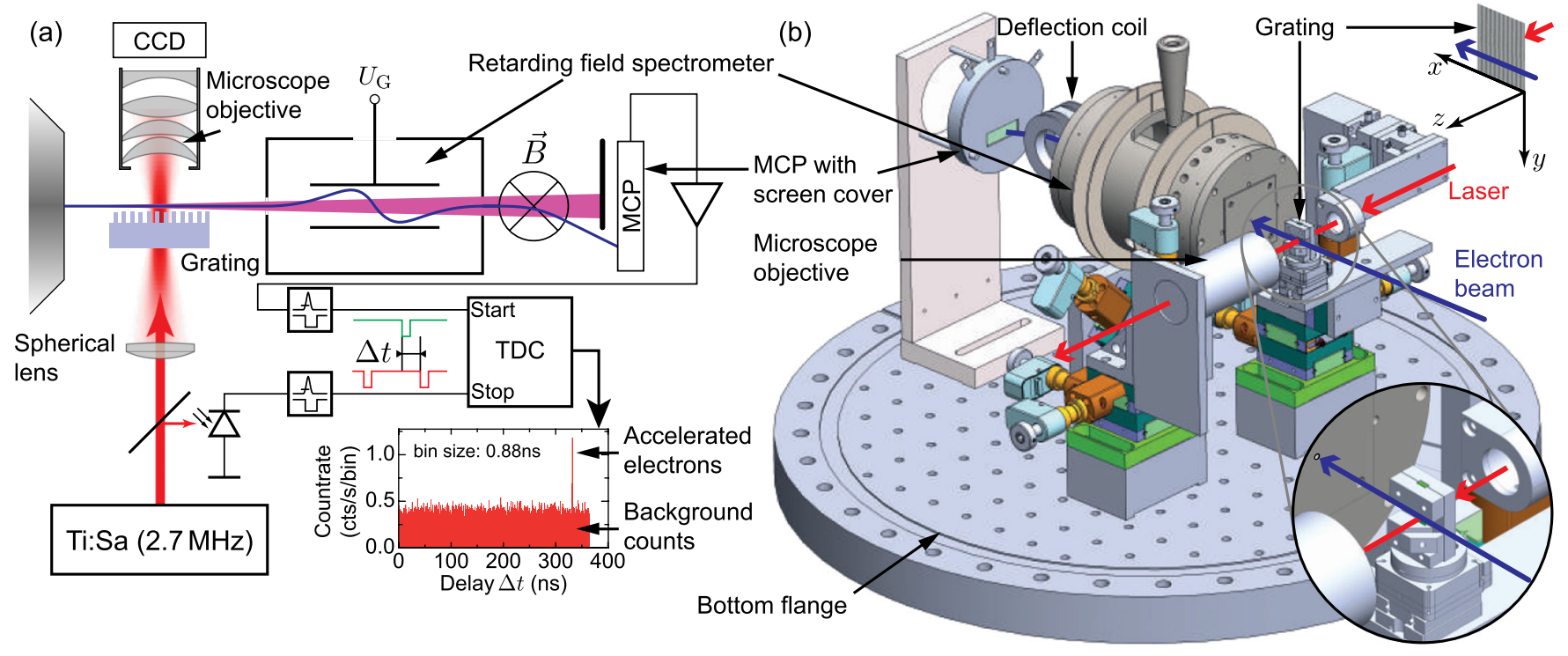

FIG. 2 (color online). (a) Conceptual picture of the electron acceleration detection. Electrons (blue trajectory) are emitted from an electron column (left) and interact with the laser pulses (red line), derived from a Titanium:sapphire oscillator, at the fused silica grating. A microscope objective is used to monitor the position of the laser focus. The electrons pass through a retarding field spectrometer, which blocks all unaccelerated electrons (counter voltage $U_{\mathrm{G}}$ ), and are detected with a MCP. The trajectory entering the spectrometer is drawn as slightly offcenter to illustrate the effect of the retarding field. A magnetic field $\vec{B}$ deflects the electrons around an x-ray beam stop to separate them from high energy photons originating inside the electron column. A time-to-digital converter (TDC) is used to measure the time delay $\Delta t$ between a detector event and the following laser pulse. This way, a signal of accelerated electrons appears at a fixed $\Delta t$ in a histogram while background counts are distributed equally over all delays (inset). (b) Technical drawing of the experimental setup with zoom-in of the grating and interaction region. The experiment is placed inside a vacuum chamber (base flange size DN $350 \mathrm{CF}$ ) evacuated to $\sim 5 \times 10^{-7}$ mbar. The microscope objective ( $x y z$-degrees of freedom), the spectrometer ( $y z$-degrees of freedom) and the grating mount ( $x y z$-degrees of freedom) can be positioned relatively to the electron beam with motorized translation stages. Additionally, the grating can be fine positioned with stick-slip piezoelectric actuators (translation in $z$-direction and rotation in $x z$-plane). The achromatic lens which is used to focus the laser pulses can be manually positioned with a compact dovetail linear stage.

increased to stay in phase with the accelerating electrons. Otherwise, without changing the grating period, dephasing limits the distance over which electrons can be accelerated. From our calculations we infer that this acceleration distance is about $25 \mu \mathrm{m}$ for acceleration gradients below $25 \mathrm{MeV} / \mathrm{m}$ for $30 \mathrm{keV}$ electrons $(\beta=0.33)$. Extensive simulation results and implications are reported in [29].

\section{EXPERIMENTAL SETUP}

\section{A. Overview}

Two schematic overviews of the experimental setup are shown in Fig. 2. The electron beam passes through the interaction region where it interacts with the evanescent field excited at the grating surface by laser pulses impinging from behind. The electrons enter a retarding field spectrometer. Accelerated electrons that pass the spectrometer are deflected by a magnetic field and are detected with a microchannel plate detector (MCP). The beam is deflected to reduce the background signal at the detector, as will be discussed below. A camera, placed outside the vacuum chamber, can be used to observe the MCP counts.

We use a Titanium:sapphire long-cavity oscillator with a repetition rate $f_{\text {rep }}=2.7 \mathrm{MHz}$, a center wavelength $\lambda=787 \mathrm{~nm}$, a pulse duration $\tau_{\mathrm{p}}=110 \mathrm{fs}$, and a pulse energy of $160 \mathrm{~nJ}$ ( $450 \mathrm{~mW}$ average output power) as the laser source [30]. A typical spectrum is shown in Fig. 3. The laser pulses are focused with an achromatic spherical lens with focal length of $30 \mathrm{~mm}$ onto the grating with a focal waist radius $w_{1}$ of $(9.0 \pm 0.4) \mu \mathrm{m}$. A microscope objective is used to monitor the laser focal spot size and position relative to the grating and electron beam and is also placed inside the vacuum chamber.

\section{B. Grating}

The fused silica grating [Figs. 1(d) and 1(e)] has been manufactured using electron beam and laser lithography in combination with reactive ion etching. With the laser wavelength of $787 \mathrm{~nm}$, a grating period of $250 \mathrm{~nm}$ is needed to accelerate $E_{\text {kin }}=27.9 \mathrm{keV}$ electrons $(\beta=0.32)$ using the first spatial harmonic. Because of a lower bound of $\lambda_{\mathrm{p}} \gtrsim 600 \mathrm{~nm}$ set by the manufacturer [31], we chose a grating period of $\lambda_{\mathrm{p}}=750 \mathrm{~nm}$ and hence use the third spatial harmonic, as depicted in Figs. 1 $(\mathrm{a}-\mathrm{c})$. The grating is located on top of a mesa (size: $2 \mathrm{~mm} \times 35 \mu \mathrm{m} \times 20 \mu \mathrm{m}$ ) that sits on top of the substrate (size: $3 \mathrm{~mm} \times$ $20 \mathrm{~mm} \times 1 \mathrm{~mm}$ ). This mesa structure allows spatial access to the grating and therefore the electron beam focus can 


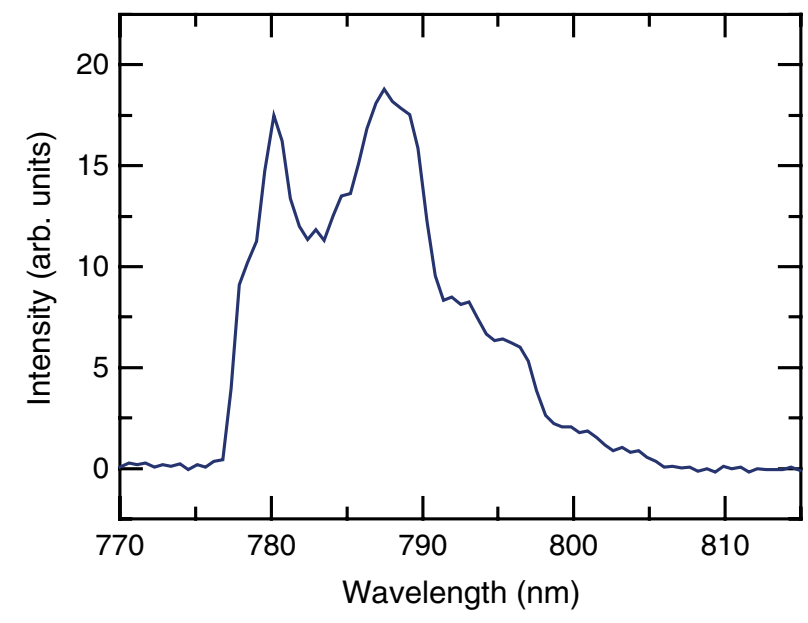

FIG. 3 (color online). Typical laser spectrum of the Ti:Sa oscillator with central wavelength $\lambda=787 \mathrm{~nm}$. The corresponding pulse duration is $\tau_{\mathrm{p}}=110 \mathrm{fs}$, measured with an autocorrelator.

approach the grating surface closer than $100 \mathrm{~nm}$ without beam clipping at the glass substrate, which would otherwise lead to surface charging and deflection of the beam.

To further reduce surface charging we first coated the sample (i.e., grating and substrate) with a $3 \mathrm{~nm}$ thick titanium layer. Afterwards we masked the mesa with a mechanical shadow mask and coated the surrounding substrate with a $10 \mathrm{~nm}$ gold layer. In a first attempt we coated the grating with gold as well, but laser irradiation melted the gold coating and formed a grained layer [Fig. 4(a)], which prevented approaching the grating surface closer than $\sim 200 \mathrm{~nm}$. Although the titanium layer is sublimated locally by the laser pulses, it does not form nanoparticles. We observe charging of the grating surface where the titanium is locally ablated as indicated by electron beam deflection, which occurs when clipping the electron beam with the mesa. But laser irradiation of the grating reduces the surface charging, which we infer from reduced distortion and deflection of the electron beam as observed from the MCP detector. We assume that this can be explained by multiphoton and avalanche ionization of the fused silica induced by the laser pulses, which increases the surface conductivity. Stuart et al. have reported an electron density of up to $10^{20} \mathrm{~cm}^{-3}$ for laser parameters $\left(I_{\mathrm{p}}=1.2 \times 10^{13} \mathrm{~W} / \mathrm{cm}^{2}, \tau_{\mathrm{p}}=100 \mathrm{fs}\right)$, which are comparable to our experiment [32].

A damage threshold measurement of the gold-coated fused silica grating is shown in Fig. 4. We observe damage for a critical peak fluence of $0.5 \mathrm{~J} / \mathrm{cm}^{2}$ and a critical peak intensity of $1.5 \times 10^{13} \mathrm{~W} / \mathrm{cm}^{2}$ [33]. The measured damage threshold fluence is a factor of 3 smaller than reported in [34]. This discrepancy between our measurement and the reported damage threshold can be partially explained by the shorter laser pulse duration as compared with the previous measurement, in which 1 ps pulses have been used [34]. We
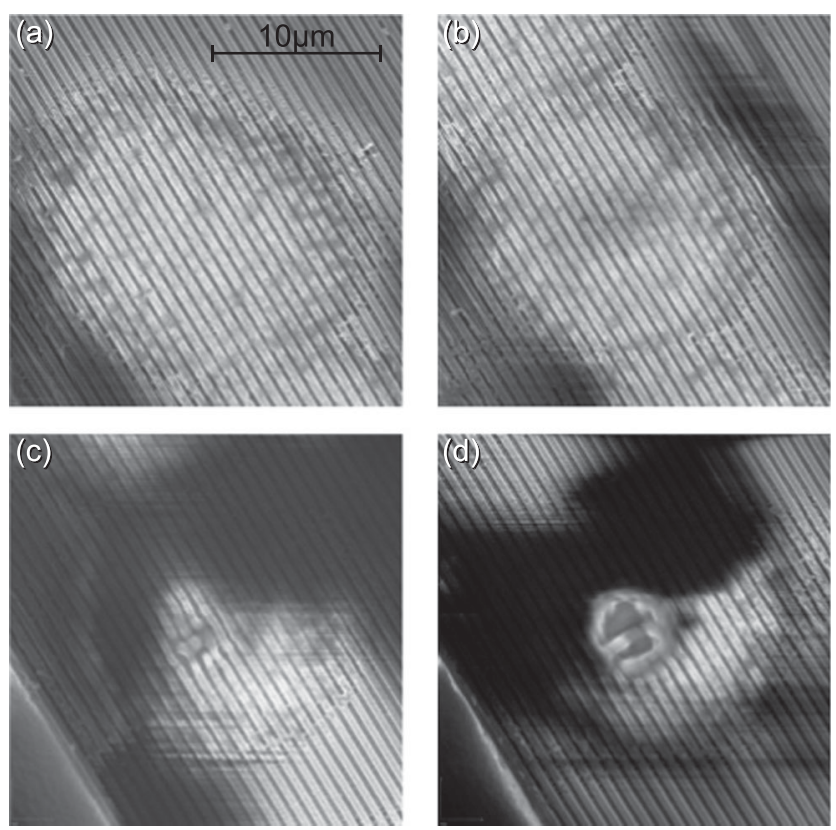

FIG. 4. Laser damage threshold measurement of a fused silica grating (10 nm gold coated) performed under high vacuum $\left(\sim 10^{-6}\right.$ mbar). Shown are scanning electron microscope images of the grating after irradiation with laser pulses for less than a minute. All four measurements are performed at different locations of the grating, which instantly experienced the full laser power, i.e., there was no slow ramp up of the power. (a) Ablation of gold coating and formation of gold nanoparticles around the laser focus. Laser parameters: average power $P=400 \mathrm{~mW}$, peak electric field $E_{\mathrm{p}}=6.1 \mathrm{GV} / \mathrm{m}$, peak intensity $I_{\mathrm{p}}=1.0 \times 10^{13} \mathrm{~W} / \mathrm{cm}^{2}$, peak fluence $F_{\mathrm{p}}=0.37 \mathrm{~J} / \mathrm{cm}^{2}$. (b) Small visible distortion of the grating in the beam center. Laser parameters: $P=500 \mathrm{~mW}, \quad E_{\mathrm{p}}=6.9 \mathrm{GV} / \mathrm{m}$, $I_{\mathrm{p}}=1.3 \times 10^{13} \mathrm{~W} / \mathrm{cm}^{2}, F_{\mathrm{p}}=0.47 \mathrm{~J} / \mathrm{cm}^{2}$. (c) Visible strong damage to the grating. Laser parameters: $P=550 \mathrm{~mW}$, $E_{\mathrm{p}}=7.2 \mathrm{GV} / \mathrm{m}, \quad I_{\mathrm{p}}=1.4 \times 10^{13} \mathrm{~W} / \mathrm{cm}^{2}, \quad F_{\mathrm{p}}=0.51 \mathrm{~J} / \mathrm{cm}^{2}$. (d) Melting of fused silica. Laser parameters: $P=620 \mathrm{~mW}$, $E_{\mathrm{p}}=7.6 \mathrm{GV} / \mathrm{m}, \quad I_{\mathrm{p}}=1.5 \times 10^{13} \mathrm{~W} / \mathrm{cm}^{2}, \quad F_{\mathrm{p}}=0.57 \mathrm{~J} / \mathrm{cm}^{2}$. The large dark areas in (c) and (d) indicate surface charging.

have applied the simple model given in [4,32], which describes the temporal evolution of the electron density $n(t)$ via

$$
\frac{d n(t)}{d t}=\alpha I(t) n(t)+\sigma_{6}[I(t)]^{6},
$$

with the laser intensity $I(t)=I_{\mathrm{p}} \exp \left[-\ln 2\left(2 t / \tau_{\mathrm{p}}\right)^{2}\right]$, the avalanche coefficient $\alpha=(4 \pm 0.6) \mathrm{cm}^{2} / \mathrm{J}$, and $\sigma_{6}=6 \times$ $10^{8 \pm 0.9} \mathrm{ps}^{-1} \mathrm{~cm}^{-3}\left(\mathrm{~cm}^{2} / \mathrm{TW}\right)^{6}$ the six-photon absorption cross section. By numerically integrating this rate equation for various laser peak intensities $I_{\mathrm{p}}$ we obtain a damage threshold intensity of $\sim(4.8 \pm 1.0) \times 10^{13} \mathrm{~W} / \mathrm{cm}^{2}$, which is defined as the intensity for which $n$ exceeds the critical density $n_{\mathrm{cr}}=10^{21} \mathrm{~cm}^{-3}$. We note that this model applies to the damage of bulk material; field enhancement at the grating surface reduces the damage threshold by about a 


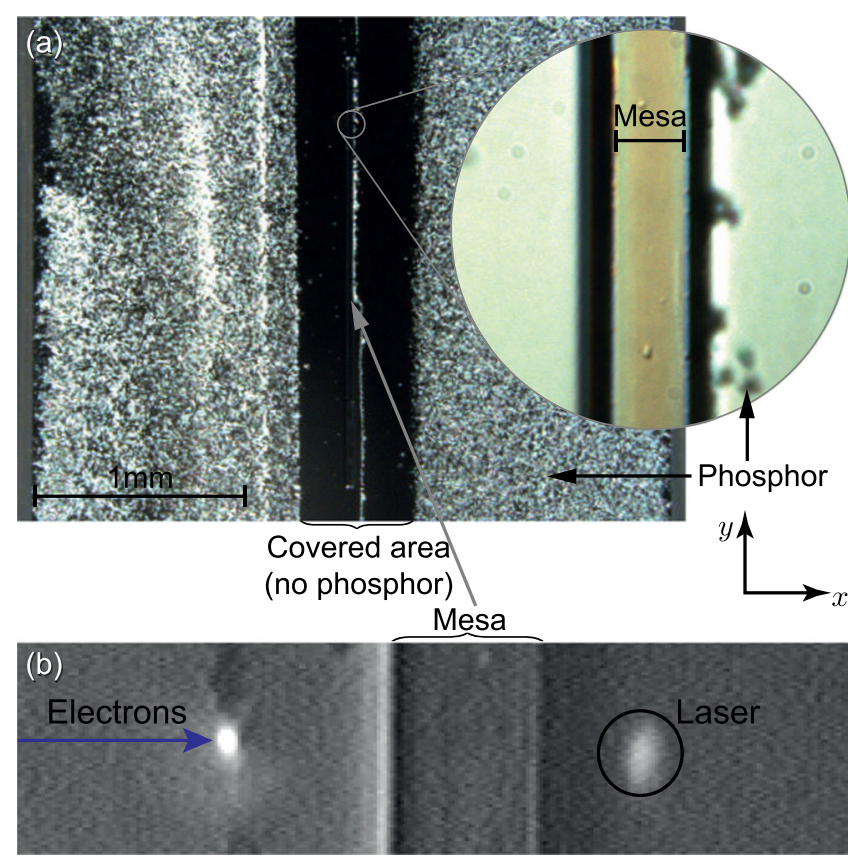

FIG. 5 (color online). (a) Microscope image of the phosphorcoated grating with closeup of the mesa. The phosphor (white grains) covers the substrate except for a $0.5 \mathrm{~mm}$ wide area around the mesa, which was masked during the coating procedure. For coarse alignment of the laser focus we use phosphor grains that have by chance accumulated right next to the mesa inside the otherwise phosphor-free region. (b) Image of the phosphorcoated grating (mesa in the center) in the experiment recorded with the microscope objective (Fig. 2). A single grain of phosphor is illuminated by the electron beam (white spot, left). We vertically overlap the laser focus (right). In this alignment procedure the grating is slightly tilted around the $y$ axis, such that the electron beam can approach the bottom of the mesa structure without being clipped at the edge of the substrate.

factor of 2 [34]. The resulting grating damage threshold intensity of $\sim(2.4 \pm 0.5) \times 10^{13} \mathrm{~W} / \mathrm{cm}^{2}$ is still slightly larger than the measured $\sim 1.5 \times 10^{13} \mathrm{~W} / \mathrm{cm}^{2}$. We attribute this minor disagreement to cumulative heating of the fused silica, which is likely to occur at $\mathrm{MHz}$ repetition rates and did not contribute in the previous studies performed at $\mathrm{kHz}$ repetition rates and below [4,32].

We coated a grating substrate with fine grain phosphor [35] to measure the overlap between the laser focus and the electron beam above the grating surface [Fig. 5(a)]. The median phosphor grain size is $2.5 \mu \mathrm{m}$, much smaller than the mesa height. Here, the mesa was covered with a mask to keep the grating clear.

In the experiment we first align the axis of the spectrometer, which is located on a translation stage (Fig. 2), with the axis of the electron column. This is done by operating the column in scanning electron microscope (SEM) imaging mode and centering the spectrometer entrance within the scanning area of the column. Similarly, we position the grating parallel and closely (within $\sim 1 \mu \mathrm{m}$ ) to the electron beam by rotating and translating the grating while observing the live SEM image.

From now on the electron beam is fixed in space by turning off the scanning mode of the SEM. The laser focus, monitored by the microscope objective, is then overlapped vertically with the beam [Fig. 5(b)] and horizontally with the mesa. Once the coarse alignment between the electron beam and the laser focus using the phosphor-coated grating has been found, fine adjustment is achieved by monitoring the electron beam deflection (i.e., beam walk off on a second time scale) while moving the edge of the mesa structure into the beam. Good overlap is indicated by minimal deflection, because laser irradiation reduces surface charging, as discussed above. We can exchange the grating without need for another coarse alignment and only have to perform a fine alignment.

\section{Electron source and spectrometer}

The electrons are derived from a conventional scanning electron microscope column (Hitachi S-570) providing energies of up to $30 \mathrm{keV}$. The energy width for an electron energy of $28 \mathrm{keV}$ is $\sim 10 \mathrm{eV}$ [Fig. 6(a)]. For this measurement we have used the retarding field spectrometer, discussed below, in combination with a switch mode power supply that is specified to provide an output voltage with residual ripple below $10 \mathrm{~V}$. Therefore the measured $10 \mathrm{eV}$ energy width represents an upper limit for the actual spectral width of the electron beam.

At a working distance of $25 \mathrm{~mm}$, the $1 / e$ focal waist radius of the electron beam is $w_{\mathrm{e}}=(70 \pm 20) \mathrm{nm}$, as inferred from knife-edge measurements [Fig. 6(b)], and the dc beam current is $I_{\mathrm{b}}=4.2 \pm 0.5 \mathrm{pA}$. We measure an opening angle of $(1.29 \pm 0.04)$ mrad implying a "Rayleigh length," that is the distance along the beam axis from the focus to the place where the beam radius is $\sqrt{2} w_{\mathrm{e}}$, of $(58 \pm 12) \mu \mathrm{m}$.

Because of the continuous-wave nature of the electron beam, only a small fraction of electrons interacts with the laser pulses: the effective electron current that can interact with the laser light is given by $I_{\text {eff }} \approx I_{\mathrm{b}} \tau_{\mathrm{p}} f_{\text {rep }}=10$ electrons per second. However, the excellent beam control of a standard SEM (small beam waist, scanning and imaging ability) together with the near unity detection efficiency of single electrons in this energy range outweigh the low expected count rate in this proof-of-concept experiment. A pulsed electron source synchronized with the laser repetition rate, which is planned to be implemented in future experiments, is expected to increase $I_{\text {eff }}$ by many orders of magnitude; 3 A peak current has been demonstrated in laser-triggered electron sources, which is $10^{12}$ times as large as in our experiment [36]. A different design of such a pulsed source focuses on the production of short (tens of femtoseconds) electron bunches suitable for the acceleration with ultrashort laser pulses [37]. 

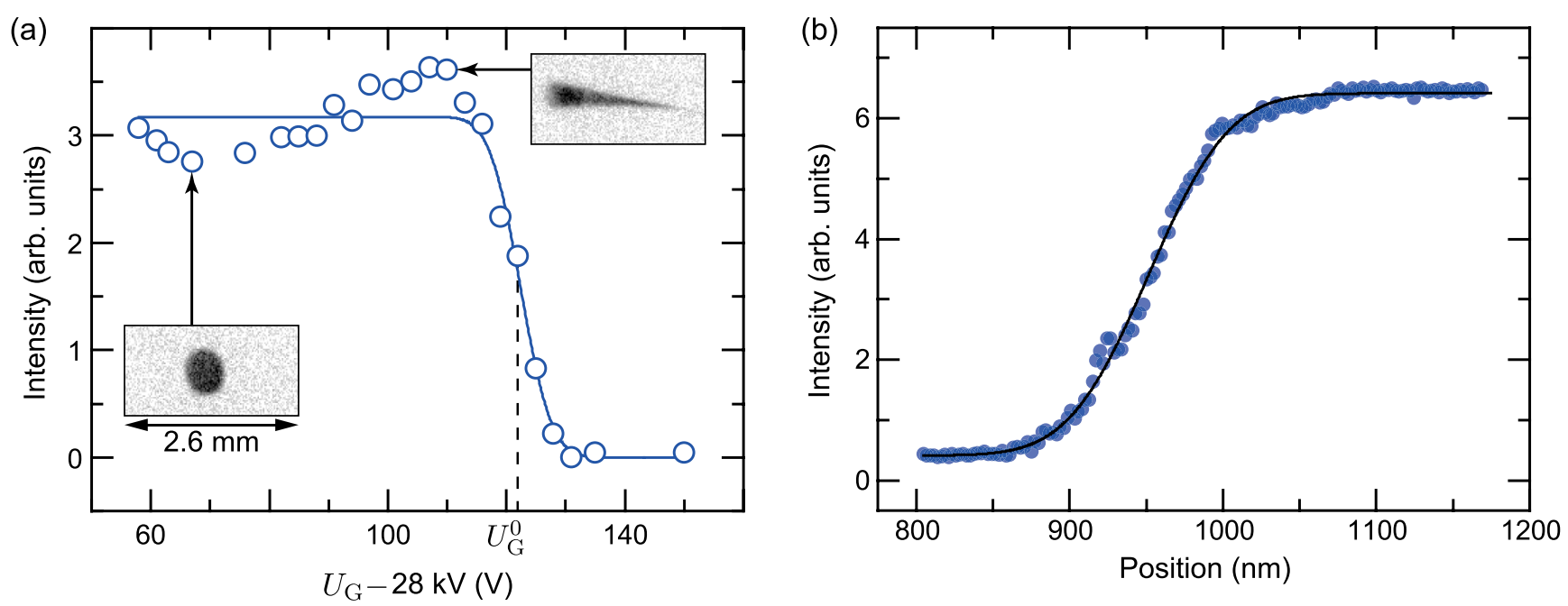

FIG. 6 (color online). (a) Integrated energy spectrum of the electron beam measured with the retarding field spectrometer shown in Fig. 7. The fitted width of the cutoff edge is $9.7 \mathrm{eV}$ (FWHM). The spectrometer center voltage is $U_{\mathrm{G}}^{0}=28122 \mathrm{~V}$ implying that for $U_{\mathrm{G}}=U_{\mathrm{G}}^{0}$ half of the dc electron beam current is blocked. The intensity in (a) and (b) has been obtained by integrating MCP images. The wavy behavior between $60 \mathrm{~V}$ and $110 \mathrm{~V}$ is due to defocusing of the electron beam with the retarding field analyzer (einzel lens). The integrated intensity appears smaller if the electron beam is more localized on the MCP. Corresponding MCP images (gray scale; white: no electrons) are shown as insets. (b) Typical measurement of the electron beam focal spot size measured by moving the edge of the mesa structure into the beam ("knife-edge method"). The fit corresponds to a $1 / e$ focal waist radius of $w_{\mathrm{e}}=58 \mathrm{~nm}$. Consecutive measurements scatter between $w_{\mathrm{e}}=50 \mathrm{~nm}$ and $w_{\mathrm{e}}=90 \mathrm{~nm}$, from which we infer $w_{\mathrm{e}}=(70 \pm 20) \mathrm{nm}$.

To measure the electron energy we use a retarding field spectrometer designed in the style of [40], shown in Figs. 7(a-c). The design of the electrode and insulator geometry as well as high-voltage conditioning has been crucial to prevent high-voltage breakdown. Breakdowns mostly originate at the metal-dielectric junction [41], which is why we removed any insulating material around the contact pin and chose a specific steplike shape for the vespel insulator [38,39]. Furthermore, the inner electrode has been mechanically polished to almost mirror quality to prevent field emission at the metal surface.

High-voltage conditioning is a well-known procedure to improve the insulation $[42,43]$. Before measurements we routinely increase the spectrometer voltage $U_{\mathrm{G}}$ starting from $\sim 20 \mathrm{kV}$ to $35 \mathrm{kV}$ in $1 \mathrm{kV}$ steps. We increase $U_{\mathrm{G}}$ by $1 \mathrm{kV}$ as soon as there are less than three high-voltage breakdown events in $20 \mathrm{~min}$, i.e., events with current spikes $\gtrsim 40 \mu \mathrm{A}$. $U_{\mathrm{G}}$ is then kept at $35 \mathrm{kV}$ at least overnight before performing a measurement. Anytime the high-voltage has been turned off this procedure has to be repeated. The whole conditioning process takes typically 12-18 hours and is computer controlled. More time is required after the initial assembly of the spectrometer, because breakdown events can originate at surface contaminations that are removed during the conditioning.

\section{Detection scheme}

In the experiment we need to measure the low expected count rate of accelerated electrons ( $I_{\text {eff }} \sim 10$ counts/s) on top of a background count rate of $\sim 50-70$ counts/s. The background originates from high-energy photons emitted from the electron column, from photoelectrons liberated by those photons and from scattered electrons coming directly from the electron column or from field-emission sites at the high-voltage connectors inside the vacuum chamber. We use a deflection coil to deflect the electron beam around an $\mathrm{x}$-ray beam stop to separate them from photons passing through the lens, as shown in Fig. 2. Without this cover in front of the MCP the background count rate would be $\sim 600$ counts/s.

In our detection scheme we measure coincidences between detector counts and laser pulses [Fig. 2(a)]. We use a microchannel plate detector to detect the electrons. We apply a voltage of $5 \mathrm{kV}$ to the phosphor screen and $\sim 2-2.5 \mathrm{kV}$ to the backside of the MCP. The front side is grounded via a bias tee and the detector counts from the bias tee's ac port are amplified and fed into a discriminator. Separately, an avalanche photodiode is used to measure the arrival time of the laser pulses picked up in front of the vacuum chamber. A time-to-digital converter measures the time difference between the detector count (start) and the next following laser pulse (stop). The signal of accelerated electrons appears at a fixed time delay in the histogram, which includes delays from 0 to $1 / f_{\text {rep }}=$ 360 ns. Background counts are uncorrelated with the laser pulses and hence distributed equally over all delays. For example, with this method we can detect 31 events in $80 \mathrm{~s}$ on top of $72 \pm 8.5$ background counts within the time delay window where the signal is located. Therefore we calculate a signal-to-noise ratio of $31 / 8.5=3.6$. 

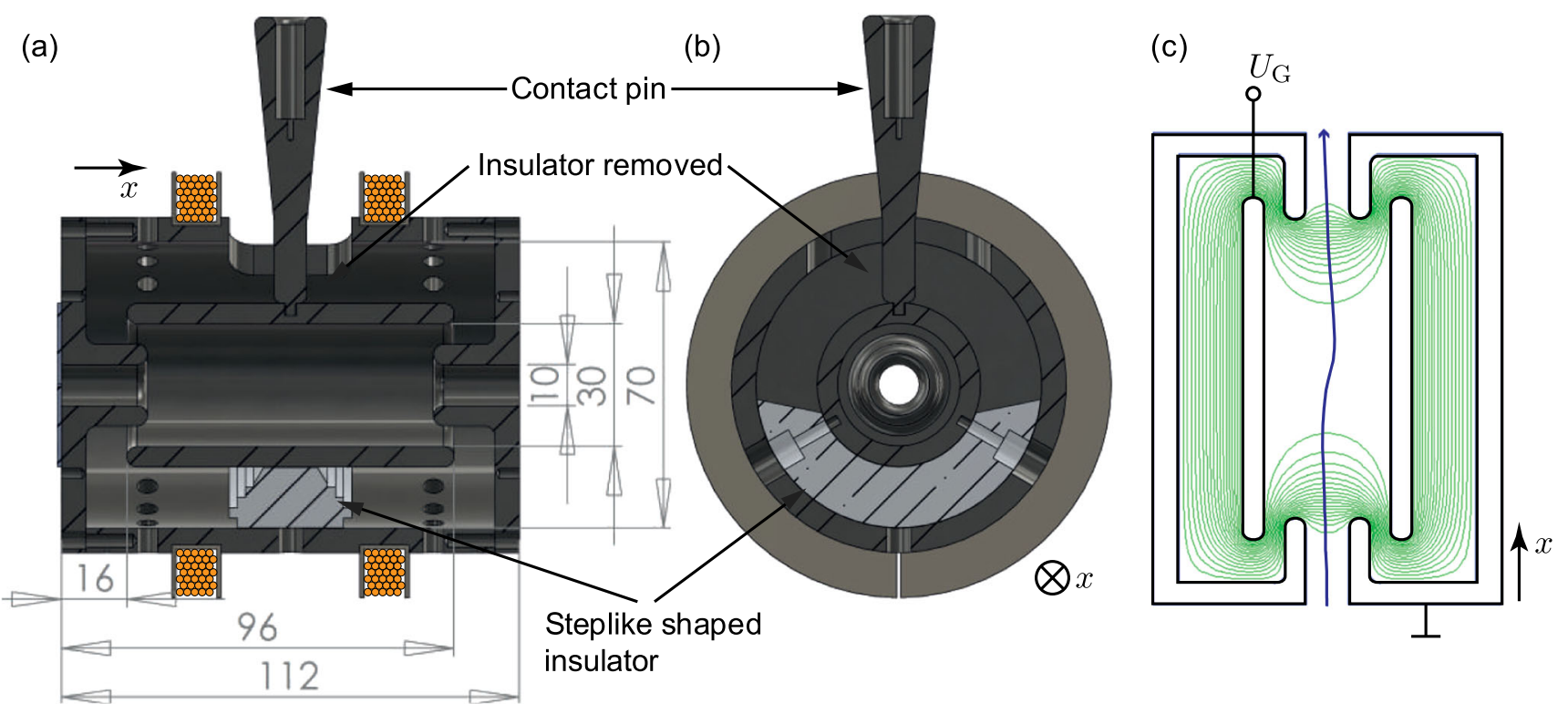

FIG. 7 (color online). (a,b) Sectional views of the electrostatic filter lens with dimensions in mm. We use stainless steel electrodes (dark gray). The vespel insulator (light gray) has been designed following the example of [38,39]. Multiple steps near the cathode collect emitted electrons, resulting in a space charge that lowers the surface electric field at the cathode and reduces electron emission [38]. The two circular $u$ profiles are coiled with wires (orange circles) to apply an axial magnetic field, which we did not use during the experiment as it distorted the electron beam focus at the grating. (c) Charged particle optics (CPO) simulation of the filter lens for $U_{\mathrm{G}}=-28.2 \mathrm{kV}$ with the electrodes (black lines), equipotential surfaces (green lines), and a sample trajectory of a $28 \mathrm{keV}$ electron (blue). From the CPO simulation we infer an offset between the applied voltage $U_{\mathrm{G}}$ and the potential barrier at the center of the filter lens of $260 \mathrm{~V}$ due to field penetration, which is why the electron still passes for the given numbers.

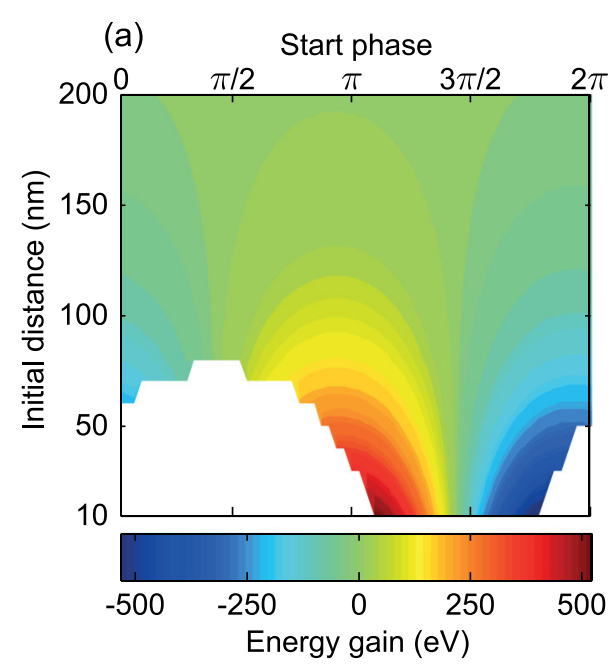

(b)

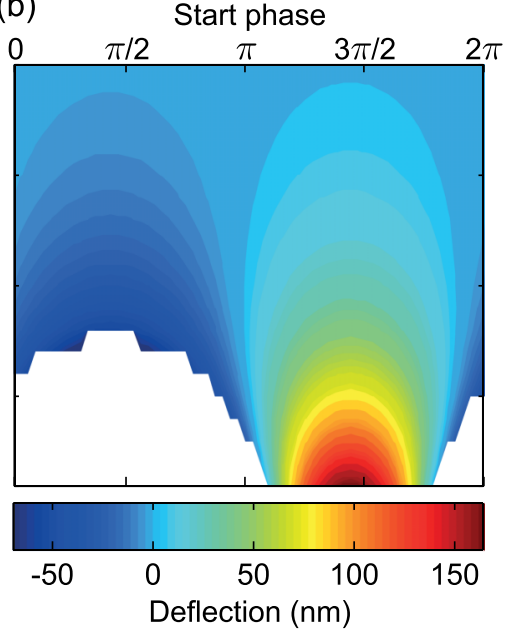

(c)

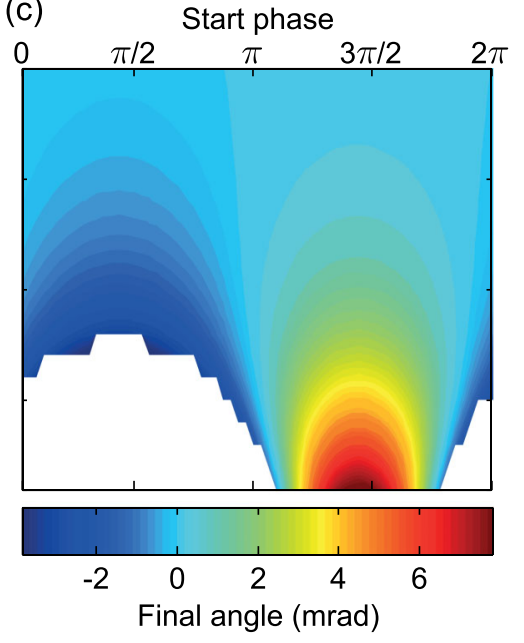

FIG. 8 (color online). Particle tracking results of a single electron interacting with the laser pulses in close proximity of the fused silica grating [Figs. 1(d) and 1(e)]. We use the experimental laser parameters mentioned in the text and a laser peak electric field of $E_{\mathrm{p}}=2.85 \mathrm{GV} / \mathrm{m}$. The initial electron energy is $E_{\mathrm{kin}}=27.9 \mathrm{keV}(\beta=0.32)$, hence electrons interact synchronously with the third spatial harmonic. Color-coded plots show the energy gain $\Delta E_{\text {kin }}$ (a), the deflection $\Delta z$ (b), and the final angle $\beta_{z} / \beta_{x}$ (c) as a function of the initial distance $z_{0}$ from the grating and the relative start phase between the electron and the laser field. For the white areas the electron crashes into the grating during the simulation. The accelerating and deflecting forces are out of phase, i.e., for the start phase with maximum acceleration the deflection vanishes and vice versa. Note that in our simulation a larger start phase corresponds to a later start time. Therefore, if electrons with a smaller start phase are accelerated less than those with a larger start phase, bunching (focusing in time) takes place. For those start phases [between $\pi / 2$ and $\pi$ in (a)] electrons become deflected towards the grating. 


\section{SIMULATION}

We use an eigenmode method [44] to simulate the excitation of spatial harmonics at the grating by a plane wave traveling perpendicularly to the grating surface [Figs. 1(a-c)]. For the grating and laser parameters mentioned above we obtain an acceleration gradient of

$$
G(z)=0.03 e E_{\mathrm{p}} \exp \left(-\frac{z}{\Gamma}\right)
$$

for synchronous acceleration using the third spatial harmonic. Here $z$ is the distance from the grating surface and $E_{\mathrm{p}}$ the laser peak electric field. This represents the maximum feasible acceleration gradient at a given distance $z$ neglecting deflection and dephasing. More details on the simulations can be found in [29].

Moreover, a particle tracking simulation is used to integrate the Lorentz force, caused by the excited near field, acting on a single electron passing the grating surface at a distance $z_{0}$ with a velocity $\beta c$. The exciting laser pulse is assumed to have an optical electric field $E_{1} \propto \exp \left[-\left(x / w_{1}\right)^{2}-2 \ln (2)\left(t / \tau_{\mathrm{p}}\right)^{2}\right] . \quad$ In the electron's moving frame the instantaneous laser electric field can be written as $E_{\mathrm{p}} \exp \left[-\left(x / w_{\text {int }}\right)^{2}\right]$, with the characteristic interaction distance

$$
w_{\text {int }}=\left(\frac{1}{w_{1}^{2}}+\frac{2 \ln (2)}{\left(\beta c \tau_{\mathrm{p}}\right)^{2}}\right)^{-1 / 2}=6.3 \mu \mathrm{m} .
$$

The results of a simulation, performed with the experimental parameters mentioned above, are shown in Fig. 8. The peak acceleration gradient can be obtained from the energy gain via

$$
G=\frac{\Delta E}{w_{\text {int }} \sqrt{\pi}} .
$$

For example, we calculate a maximum energy gain $\Delta E=$ $90 \mathrm{eV}$ at $z_{0}=100 \mathrm{~nm}$. This corresponds to a maximum acceleration gradient of $8 \mathrm{MeV} / \mathrm{m}$. The acceleration decays exponentially with increasing distance from the grating with a decay constant $\Gamma=42 \mathrm{~nm}$, as expected. It can be seen that depending on the relative position of the electron inside the laser field, the force acts accelerating, decelerating, or deflecting. Furthermore, the accelerating and deflecting forces are out of phase. Note that most of the electrons passing the grating surface at distances below $\sim 50 \mathrm{~nm}$ crash into the grating and thus cannot be detected. A minimal distance of $50 \mathrm{~nm}$ agrees well with the measured maximum acceleration gradient of $25 \mathrm{MeV} / \mathrm{m}$, discussed below.

The accelerated fraction is defined as the ratio of the number of accelerated electrons $I_{\text {acc }}$ to the number of electrons $I_{\text {eff }}$ that can interact with the laser pulse:
$\alpha=I_{\text {acc }} / I_{\text {eff }}$. To calculate $\alpha$ based on the simulation results we assume a Gaussian-shaped electron beam current density

$$
j(z)=\frac{I_{\mathrm{eff}}}{\sqrt{\pi} w_{\mathrm{e}}} e^{-\left(z-z_{0}\right)^{2} / w_{\mathrm{e}}^{2}}
$$

with the $1 / e$ electron beam waist $w_{\mathrm{e}}$ [Fig. 9(a)]. In the experiment we measure an integrated accelerated fraction $\alpha(\Delta E)$; that is, the integrated signal of electrons that are accelerated sufficiently to pass the spectrometer and therefore gain more energy than $\Delta E=e U_{\mathrm{G}}-e U_{\mathrm{G}}^{0}$. Here, $U_{\mathrm{G}}$ is the applied spectrometer voltage and $U_{\mathrm{G}}^{0}$ is the center spectrometer voltage of the energy spectrum, defined in Fig. 6(a). We derive

$$
\alpha(\Delta E)=\frac{1}{I_{\text {eff }}} \times \int_{50 \mathrm{~nm}}^{z_{\max }} \zeta(\Delta E, z) j(z) f_{\text {rep }} \tau_{\mathrm{p}} \mathrm{d} z
$$

with the differential accelerated fraction $\zeta(\Delta E, z)$ as a function of distance $z$ and energy gain $\Delta E$ [Fig. 9(b)] and the maximum distance $z_{\max }$ at which electrons gain more energy than $\Delta E$. The lower bound of the integral reflects the assumption that electrons cannot pass the grating any closer than $50 \mathrm{~nm}$. The factor $f_{\text {rep }} \tau_{\mathrm{p}}$ corresponds to the fraction of time during which the electrons can effectively interact with the laser pulses. In the end we scale the overall amplitude of $\alpha$ to fit the experimental data. $z_{\max }$ can be directly calculated by rearranging Eqs. (4) and (6):

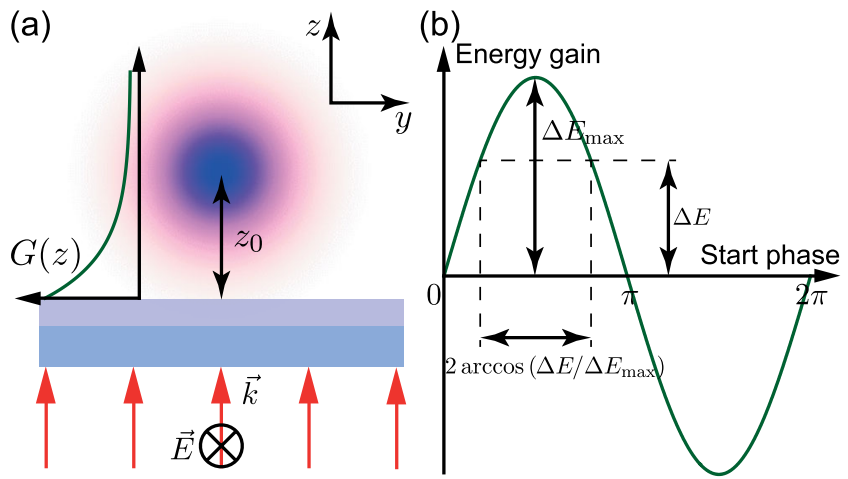

FIG. 9 (color online). (a) Side view of a dielectric grating (light blue) with an electron beam (dark blue) passing at a distance $z_{0}$. The laser is incident from below (red arrows). We assume a constant laser field over the width of the Gaussian electron beam profile $\left(w_{1} \gg w_{\mathrm{e}}\right)$ and model a 1D Gaussian current density [Eq. (7)] being subject to the acceleration gradient $G(z)=0.03 \times e E_{\mathrm{p}} \exp (-z / \Gamma)$, with $\Gamma=42 \mathrm{~nm} \quad[\mathrm{Eq}$. (2)]. (b) Energy gain as a function of start phase at a fixed distance from the grating surface [corresponding to a horizontal slice in Fig. 8(a)]. Here, we neglect deflection of the electrons. The differential accelerated fraction at this distance equals $\zeta(\Delta E, z)=\arccos \left[\Delta E / \Delta E_{\max }(z)\right] / \pi$. 


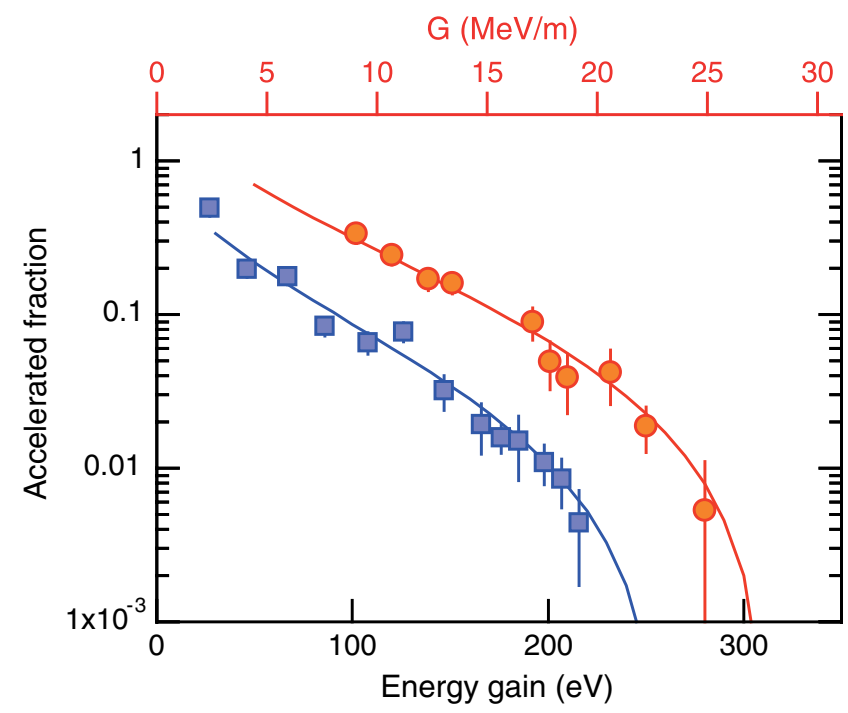

FIG. 10 (color online). Measurement of the accelerated fraction as a function of energy gain (bottom axis) and acceleration gradient (top axis) for two different laser peak electric fields $\left[E_{\mathrm{p}}=2.85 \mathrm{GV} / \mathrm{m}\right.$ (orange circles), $E_{\mathrm{p}}=2.36 \mathrm{GV} / \mathrm{m}$ (blue squares)]. We measure a maximum energy gain of $280 \mathrm{eV}$ corresponding to a maximum acceleration gradient of $25 \mathrm{MeV} / \mathrm{m}$. The curves represent simulation results, which were obtained according to Eq. (8) for $z_{0}=120 \mathrm{~nm}$ and $w_{\mathrm{e}}=77 \mathrm{~nm}$. The overall amplitude of the simulated values has been scaled to fit the experimental data.

$$
z_{\max }=\Gamma \ln \left(\frac{0.03 e E_{\mathrm{p}} \sqrt{\pi} w_{\text {int }}}{\Delta E}\right)
$$

For example, assuming $E_{\mathrm{p}}=2.85 \mathrm{GV} / \mathrm{m}$, electrons have to pass the grating within $z_{\max }=94 \mathrm{~nm}$ to gain more than $100 \mathrm{eV}$ in energy.

\section{EXPERIMENTAL RESULTS}

In this section we discuss the experimental results published in [28] in more detail.

In Fig. 10 we compare measurements of the accelerated fraction as a function of the acceleration gradient for two different laser peak electric fields of $E_{\mathrm{p}}=2.85 \mathrm{GV} / \mathrm{m}$ (average power $P=450 \mathrm{~mW}$, peak intensity $I_{\mathrm{p}}=$ $2.2 \times 10^{12} \mathrm{~W} / \mathrm{cm}^{2}$, peak fluence $\left.F_{\mathrm{p}}=0.13 \mathrm{~J} / \mathrm{cm}^{2}\right)$ and $E_{\mathrm{p}}=2.36 \mathrm{GV} / \mathrm{m}\left(P=300 \mathrm{~mW}, I_{\mathrm{p}}=1.5 \times 10^{12} \mathrm{~W} / \mathrm{cm}^{2}\right.$, $F_{\mathrm{p}}=0.09 \mathrm{~J} / \mathrm{cm}^{2}$ ) with simulation results. We observe a maximum measured energy gain of $\Delta E=280 \mathrm{eV}$. It corresponds to a maximum acceleration gradient of $G_{\max }=25 \mathrm{MeV} / \mathrm{m}$, according to Eq. (6). This is already comparable with state-of-the-art rf linacs. The simulated curves of the accelerated fraction assume a distance of the electron beam center from the grating surface of $z_{0}=$ $(120 \pm 10) \mathrm{nm}$ and an electron beam waist of $w_{\mathrm{e}}=77 \mathrm{~nm}$. We deduce from our simulations that the maximum acceleration occurs for electrons that pass the grating at a distance of $\sim 50 \mathrm{~nm}$ due to the finite beam width. We infer this to be the experimental limit for the minimum distance between the electrons and the grating. We assume that beam clipping together with residual surface charging prevents a closer approach in the current setup.

Only the component of the laser electric field that is parallel to the electrons' velocity can excite the accelerating spatial harmonic. Hence, the accelerated fraction depends on the laser polarization angle $\varphi$ between the incident laser electric field and the electrons' trajectory like $\cos (\phi)$ (Fig. 11). This measurement proves the direct acceleration with the laser field and rules out intensity-dependent but polarization-independent ponderomotive acceleration [45]. Its effect we estimate to a maximum of $G_{\text {pond }}=$ $e^{2} E_{\mathrm{p}}^{2} \exp (-0.5) /\left(2 m \omega^{2} w_{1}\right)=12 \mathrm{keV} / \mathrm{m}$, with the electron mass $m$ and the laser angular frequency $\omega=2 \pi f$.

The sinusoidal fit in Fig. 11 gives a limit on a possible angular misalignment of the grating with respect to the electron beam of $\varphi_{0}=(3.2 \pm 5.3)^{\circ}$. This angular misalignment implies an offset between the experimentally measured electron energy and the energy used in the simulations, where we set $\phi_{0}=0$, in the following way. The component of the electron velocity that is parallel to the grating vector has to satisfy the synchronicity condition and therefore $\beta \cos \left(\varphi_{0}\right)=\lambda_{\mathrm{p}} /(n \lambda)$. However, in the experiment we measure the total kinetic energy related to an electron velocity $\beta$, which is a factor of $1 / \cos \phi_{0}$ larger than the design velocity $\lambda_{\mathrm{p}} /(n \lambda)$. We calculate the resulting shift of the measured kinetic energy to be $-100_{-570}^{+100} \mathrm{eV}$.

The measurement of the accelerated fraction as a function of the relative distance $z_{0}$ between the electron

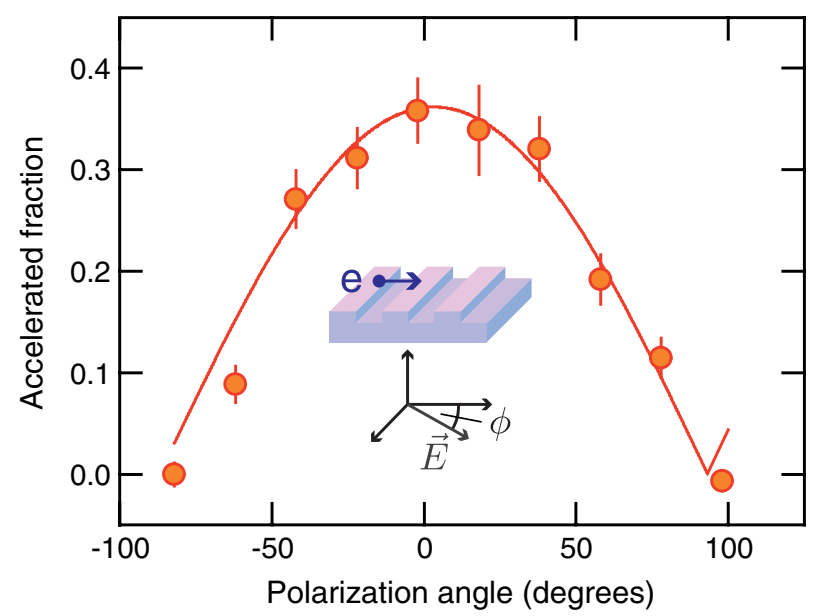

FIG. 11 (color online). Accelerated fraction of electrons as a function of the laser polarization angle $\phi$ relative to the electrons' trajectory. $\phi=0^{\circ}$ means that the laser polarization is parallel to the electrons' momentum, $\phi=90^{\circ}$ that it is perpendicular to it (see inset). The data agree well with the expected cosine behavior (orange fit curve) and proves that the electrons are directly accelerated by the light field. 


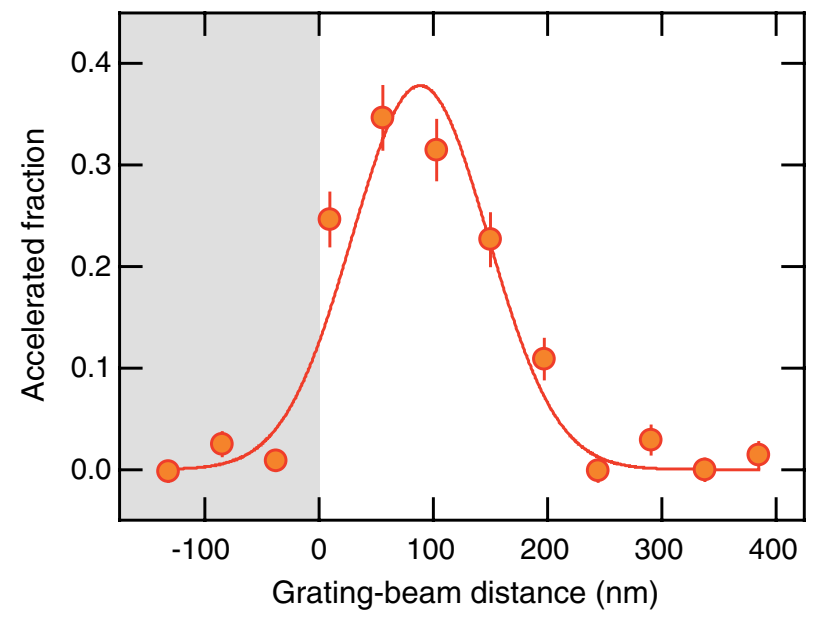

FIG. 12 (color online). Measurement of the accelerated fraction versus the distance between the grating surface (shaded area) and the electron beam center, with Gaussian fit (solid curve). This measurement has been performed with a laser peak electric field of $E_{\mathrm{p}}=2.36 \mathrm{GV} / \mathrm{m}$ and at a fixed energy gain $\Delta E=30 \mathrm{eV}$. Because of the finite width $w_{\mathrm{e}}$ of the electron beam, we measure a few accelerated electrons even when the beam center lies slightly inside the grating. The absolute position $\left(z_{0}=0\right)$ of the data relative to the grating surface has been determined from simulations [Eq. (8)].

beam and the grating surface is shown in Fig. 12. It confirms that acceleration is only possible in close vicinity of the grating surface, where the evanescent field is pronounced. The signal decreases for distances smaller than $100 \mathrm{~nm}$ due to clipping of the electron beam at the mesa structure. The data can be fitted with a Gaussian of width $(119 \pm 11) \mathrm{nm}$. From simulations of the accelerated fraction [Eq. (8)] for varying $z_{0}$ and for the experimental parameters $\left(E_{\mathrm{p}}=2.36 \mathrm{GV} / \mathrm{m}\right.$ and $\left.\Delta E=30 \mathrm{eV}\right)$ we also obtain a Gaussian dependence. The measured width of the Gaussian can be reproduced with simulation results for $w_{\mathrm{e}}=(77 \pm 8) \mathrm{nm}$, in good agreement with the experimental result $w_{\mathrm{e}}=(70 \pm 20) \mathrm{nm}$ obtained by knife-edge measurements (Fig. 6).

In Fig. 13 we compare a measurement of the maximum acceleration gradient for varying electron energy with $a b$ initio calculations. Here, we observe the largest acceleration gradient for an initial electron energy of $\sim 27.7 \mathrm{keV}$. The acceleration efficiency decreases for larger and smaller electron energies. Hence, this measurement proves that for efficient acceleration the synchronicity condition has to be fulfilled. The $a b$ initio calculations correspond to electrons passing the grating at $z_{0}=60 \mathrm{~nm}$ and show good agreement with the experiment. In order to reduce the measuring time and increase the statistics we define the maximum acceleration gradient in this measurement at a larger accelerated fraction than in Fig. 10(a) $\left(4 \times 10^{-2}\right.$ versus $5 \times 10^{-3}$ ), which explains why we measure a maximum gradient of $\sim 20 \mathrm{MeV} / \mathrm{m}$ instead of $25 \mathrm{MeV} / \mathrm{m}$.

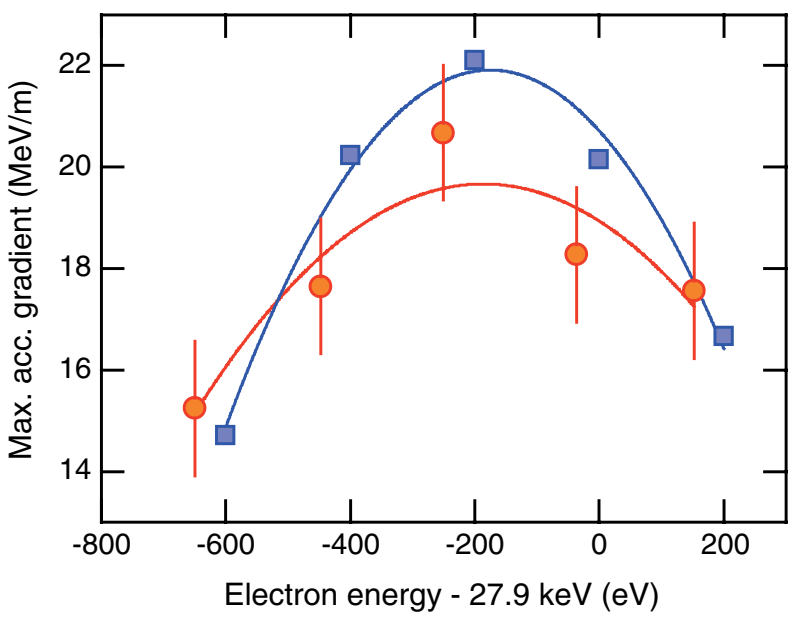

FIG. 13 (color online). Measurement (orange circles) and $a b$ initio simulation (blue squares) of the maximum acceleration gradient as a function of the initial electron energy, with quadratic fits as guides to the eye (solid lines). The largest acceleration gradient occurs $200 \mathrm{eV}$ below the synchronous energy $(27.9 \mathrm{keV})$ because the acceleration at the rising edge of the Gaussian-shaped interaction, with width $w_{\text {int }}$, ensures synchronicity during the presence of the laser peak electric field. The slight difference between the curves' width requires further investigation. The experimental values have all together been shifted by $-340 \mathrm{eV}$ to match the simulation results $(-260 \mathrm{eV}$ due to field penetration (Fig. 7) and $-100_{-570}^{+100} \mathrm{eV}$ due to angular misalignment of the grating).

\section{CONCLUSION AND OUTLOOK}

In conclusion, we have presented the important details of a proof-of-principle experiment on dielectric laser acceleration of nonrelativistic electrons at a photonic grating structure. Our results represent the first demonstration of scalable laser acceleration as well as of the inverse SmithPurcell effect in the optical regime. We have observed an acceleration gradient of $25 \mathrm{MeV} / \mathrm{m}$ and expect up to 2 orders of magnitude larger gradients for relativistic electrons. This may lead to a next generation of dielectric laser accelerators with application in, e.g., free-electron lasers (FELs), where high-brightness, low-emittance beams are required to produce coherent $\mathrm{x}$-ray radiation [46]. Dielectric laser accelerators naturally provide ultralowemittance beams due to the micron-sized beam diameter. A combination with sharp metal tips as sources of highbrightness electron beams [36,47-49] may enable the envisioned all-optical table-top FEL $[23,24]$. Next steps comprise the full simulation and realization of doublegrating structures that can accelerate electrons from a highbrightness laser-triggered source [37] up to relativistic energies and the demonstration of focusing, deflecting, and bunching structures. Other applications of nonrelativistic laser-driven acceleration structures include ultrafast electron diffraction experiments and time-resolved electron microscopy. 


\section{ACKNOWLEDGMENTS}

We gratefully acknowledge J. Hoffrogge for work on the electron column, H. Ramadas and R. Davies for simulation work, P. Altpeter for titanium coating, and the Stanford DARPA AXiS Collaboration for discussions. This work has been funded by the Max Planck Society and the DFG Cluster of Excellence Munich-Centre for Advanced Photonics.

[1] K. Shimoda, Appl. Opt. 1, 33 (1962).

[2] N. A. Solyak, AIP Conf. Proc. 1086, 365 (2009).

[3] B. Spataro, D. Alesini, V. Chimenti, V. Dolgashev, A. Haase, S. Tantawi, Y. Higashi, C. Marrelli, A. Mostacci, R. Parodi, and A. Yeremian, Nucl. Instrum. Methods Phys. Res., Sect. A 657, 114 (2011).

[4] M. Lenzner, J. Krüger, S. Sartania, Z. Cheng, C. Spielmann, G. Mourou, W. Kautek, and F. Krausz, Phys. Rev. Lett. 80, 4076 (1998).

[5] A. Baltuška, T. Udem, M. Uiberacker, M. Hentschel, E. Goulielmakis, C. Gohle, R. Holzwarth, V. S. Yakovlev, A. Scrinzi, T. W. Hänsch, and F. Krausz, Nature (London) 421, 611 (2003).

[6] J. Rosenzweig, A. Murokh, and C. Pellegrini, Phys. Rev. Lett. 74, 2467 (1995).

[7] Y. C. Huang, D. Zheng, W. M. Tulloch, and R. L. Byer, Appl. Phys. Lett. 68, 753 (1996).

[8] R. B. Yoder and J. B. Rosenzweig, Phys. Rev. ST Accel. Beams 8, 111301 (2005).

[9] T. Plettner, P. P. Lu, and R. L. Byer, Phys. Rev. ST Accel. Beams 9, 111301 (2006).

[10] B. M. Cowan, Phys. Rev. STAccel. Beams 11, 011301 (2008).

[11] B. Naranjo, A. Valloni, S. Putterman, and J. B. Rosenzweig, Phys. Rev. Lett. 109, 164803 (2012).

[12] S. Payeur, S. Fourmaux, B. E. Schmidt, J. P. MacLean, C. Tchervenkov, F. Legare, M. Piche, and J. C. Kieffer, Appl. Phys. Lett. 101, 041105 (2012).

[13] M. O. Scully and M. S. Zubairy, Phys. Rev. A 44, 2656 (1991).

[14] E. Esarey, P. Sprangle, and J. Krall, Phys. Rev. E 52, 5443 (1995).

[15] E. Esarey, C. B. Schroeder, and W. P. Leemans, Rev. Mod. Phys. 81, 1229 (2009).

[16] T. Plettner, R. L. Byer, E. Colby, B. Cowan, C. M. S. Sears, J. E. Spencer, and R. H. Siemann, Phys. Rev. Lett. 95, 134801 (2005).

[17] T. Plettner, R. L. Byer, E. Colby, B. Cowan, C. M. S. Sears, J. E. Spencer, and R. H. Siemann, Phys. Rev. ST Accel. Beams 8, 121301 (2005).

[18] Y. Takeda and I. Matsui, Nucl. Instrum. Methods 62, 306 (1968).

[19] R. Palmer, Part. Accel. 11, 81 (1980).

[20] K. Mizuno, S. Ono, and O. Shimoe, Nature (London) 253, 184 (1975).

[21] K. Mizuno, J. Pae, T. Nozokido, and K. Furuya, Nature (London) 328, 45 (1987).

[22] J. Bae, H. Shirai, T. Nishida, T. Nozokido, K. Furuya, and K. Mizuno, Appl. Phys. Lett. 61, 870 (1992).
[23] T. Plettner and R. L. Byer, Phys. Rev. ST Accel. Beams 11, 030704 (2008).

[24] T. Plettner and R. L. Byer, Nucl. Instrum. Methods Phys. Res., Sect. A 593, 63 (2008).

[25] T. Plettner, R. L. Byer, C. McGuinness, and P. Hommelhoff, Phys. Rev. ST Accel. Beams 12, 101302 (2009).

[26] T. Plettner, R. L. Byer, and B. Montazeri, J. Mod. Opt. 58, 1518 (2011).

[27] E. A. Peralta, K. Soong, R. J. England, E. R. Colby, Z. Wu, B. Montazeri, C. McGuinness, J. McNeur, K. J. Leedle, D. Walz, E. B. Sozer, B. Cowan, B. Schwartz, G. Travish, and R. L. Byer, Nature (London) 503, 91 (2013).

[28] J. Breuer and P. Hommelhoff, Phys. Rev. Lett. 111, 134803 (2013).

[29] J. Breuer and P. Hommelhoff (to be published).

[30] S. Naumov, A. Fernandez, R. Graf, P. Dombi, F. Krausz, and A. Apolonski, New J. Phys. 7, 216 (2005).

[31] Fraunhofer Institute for Applied Optics and Precision Engineering, Jena, Germany.

[32] B. C. Stuart, M. D. Feit, S. Herman, A. M. Rubenchik, B. W. Shore, and M. D. Perry, J. Opt. Soc. Am. B 13, 459 (1996).

[33] For this measurement we use a tighter laser focus of $5 \mu \mathrm{m}$ and shorter 70 fs pulses. However, in this short-pulse regime the laser spectrum was unstable and mode-locking could not be maintained for more than a couple of minutes. That is why the main experiment was conducted with longer $110 \mathrm{fs}$ pulses.

[34] K. Soong, R. L. Byer, C. McGuinness, E. Peralta, and E. Colby, in Proceedings of the 24th Particle Accelerator Conference, PAC-2011 (IEEE, New York, 2011), pp. 277-279.

[35] P43 phosphor UKL65/UF-C1 from Phosphor Technology Ltd.

[36] R. Ganter, R. Bakker, C. Gough, S. C. Leemann, M. Paraliev, M. Pedrozzi, F. Le Pimpec, V. Schlott, L. Rivkin, and A. Wrulich, Phys. Rev. Lett. 100, 064801 (2008).

[37] J. Hoffrogge, J.-P. Stein, M. Krüger, M. Förster, J. Hammer, D. Ehberger, P. Baum, and P. Hommelhoff, arXiv:1303.2383.

[38] J. P. Shannon, S. F. Philp, and J. G. Trump, J. Vac. Sci. Technol. 2, 234 (1965).

[39] R. Hawley, Vacuum 18, 383 (1968).

[40] K. Brack, Z. Naturforsch. Teil A 17, 1066 (1962).

[41] M. J. Kofoid, Trans. Am. Inst. Electr. Eng., Part 3 79, 991 (1960).

[42] G. F. Steib and E. Moll, J. Phys. D 6, 243 (1973).

[43] W. T. Diamond, J. Vac. Sci. Technol. A 16, 720 (1998).

[44] D. M. Pai and K. A. Awada, J. Opt. Soc. Am. A 8, 755 (1991).

[45] H. A. H. Boot and R. B. R.-S.-Harvie, Nature (London) 180, 1187 (1957).

[46] B. W. J. McNeil and N. R. Thompson, Nat. Photonics 4, 814 (2010).

[47] P. Hommelhoff, Y. Sortais, A. Aghajani-Talesh, and M. A. Kasevich, Phys. Rev. Lett. 96, 077401 (2006).

[48] S. Tsujino, P. Beaud, E. Kirk, T. Vogel, H. Sehr, J. Gobrecht, and A. Wrulich, Appl. Phys. Lett. 92, 193501 (2008).

[49] A. Mustonen, P. Beaud, E. Kirk, T. Feurer, and S. Tsujino, Appl. Phys. Lett. 99, 103504 (2011). 